\title{
Valle Giumentina (Abruzzes, Italie)
}

Troisième mission : étude des comportements techno-économiques au Pléistocène moyen

\section{Élisa Nicoud, Daniele Aureli et Marina Pagli}

\section{(2) OpenEdition}

\section{Journals}

Édition électronique

URL : http://journals.openedition.org/cefr/1306

DOI : $10.4000 /$ cefr. 1306

ISSN : 2282-5703

Éditeur

École française de Rome

Référence électronique

Élisa Nicoud, Daniele Aureli et Marina Pagli, «Valle Giumentina (Abruzzes, Italie) », Chronique des activités archéologiques de l'École française de Rome [En ligne], Italie centrale, mis en ligne le 06 février 2015, consulté le 19 avril 2019. URL : http://journals.openedition.org/cefr/1306 ; DOI : 10.4000/ cefr.1306

Ce document a été généré automatiquement le 19 avril 2019

(c) École française de Rome 


\title{
Valle Giumentina (Abruzzes, Italie)
}

Troisième mission : étude des comportements techno-économiques au Pléistocène moyen

\author{
Élisa Nicoud, Daniele Aureli et Marina Pagli
}

\section{NOTE DE L'AUTEUR}

Les chercheurs impliqués dans le projet couvrent de nombreuses disciplines de l'étude du Quaternaire. Les chercheurs dont le nom est suivi d'un astérisque ont contribué directement à ce rapport : Silvano Agostini, Giovanni Boschian, Jean-Jacques Bahain, Christine Chaussé, Jean-Philippe Degeai*, Biagio Giaccio, Fabio Fusco*, Marion Hernandez, Catherine Kuzucuoglu, Christelle Lahaye, Cristina Lemorini, Nicole Limondin-Lozouet*, Paolo Mazza*, Norbert Mercier, Sébastien Nomade, Alison Peirera, Vincent Robert, Maria Adelaide Rossi*, Valentina Villa, Clément Virmoux et Andrea Zupancich.

Les bénévoles étaient Français, Italiens et Brésilien, issus de nombreuses institutions : EFR, Ambassade de France en Italie, des Universités de Chieti-Pescara, Roma-La Sapienza, Napoli L'Orientale, Venezia Ca' Foscari, Pisa, Bordeaux-Montaigne, Madrid Complutense, Aix-Marseille, Paris 1 Panthéon-Sorbonne, Lyon 2, Rennes, Muséum national d'Histoire naturelle et de la commune d'Abbateggio.

Il s'agit de : Lourdeau Antoine (MCF MNHN), Rocca Roxane (docteur UMR 7041), Di Marco Mattia, Zaminga Walter, Berilli Lorenzo, Joseph Di Pierdomenico, Tonino Leardi, Camillo Simone (Abbateggio), Palmerini Guido, Bottaro Elena, Martin Cristina, Magliocchi Andrea, Bruschini Marta, Dias da Silva Vanessa, Campeti Silvia, Carpentieri Marco, Di Matteo Martina, Guidi Lorenzo, Mulargia Marco, Abruzzese Claudia, D’Alonzo Francesca, D'Itria Elena, Fusco Marianna, Monaco Serena, Bosq Valérie, Capellari Félicien, Bardey Vaillant Morgane, Durocher Marine, Marandas Lucie, Robin Agapé, Roth Léa, Floury Emilie, Rousselot Lauren, Barrier Alexandre, Battistelli Matthieu, Einhorn Camille, Franchi Hélène. 


\section{Les objectifs de la mission de terrain 2014}

1 Valle Giumentina est un gisement des Abruzzes du Paléolithique ancien et moyen en plein air, fouillé brièvement dans les années 1950 par le professeur A. M. Radmilli de l'Université de Pise, en collaboration avec le géologue J. Demangeot. Sept horizons archéologiques ont été mis au jour en contexte globalement lacustre, à la faveur d'une ravine profonde qui a incisé les dépôts du pléistocène jusqu’à $25 \mathrm{~m}$ de profondeur ${ }^{1}$.

2 Dans le cadre du programme quinquennal de l'École française de Rome (2012-2016), les recherches pluridisciplinaires ont repris sur le terrain en collaboration avec la Soprintendenza per $i$ beni archeologici dell'Abruzzo afin de préciser la chronostratigraphie du gisement, de déterminer la nature technique des industries lithiques présentes et les modalités d'occupation du site. Il s'agit à terme, d'insérer ces données renouvelées dans le contexte régional, national et européen du Paléolithique ancien et moyen, puisque Valle Giumentina est depuis sa découverte, considéré comme un gisement de référence à chacune de ces échelles.

3 Après deux missions essentiellement consacrées à l'obtention de données chronostratigraphiques et géomorphologiques, durant lesquelles une coupe stratigraphique de $16,40 \mathrm{~m}$ de hauteur a été mise au jour, un carottage mécanique réalisé jusqu'à $45 \mathrm{~m}$ de profondeur, et des sondages géophysiques effectués en différents axes de la vallée, la troisième mission a permis quant à elle, d'obtenir de nombreuses données archéologiques.

4 En 2014, la mission principale a duré 45 jours, du 18 mai au 1 juillet. En raison du mauvais temps, les reconnaissances géomorphologiques ont été reportées à la dernière semaine d'août. Les activités à visée archéologique et géochronologique se sont inscrites pleinement dans la continuité de celles de l'an passé. La fouille planimétrique a été poursuivie et l'aire agrandie; différentes activités avaient trait à la définition du cadre chronostratigraphique et paléoenvironnemental et plusieurs jours ont été consacrés à l'appréhension de l'évolution géomorphologique du site (figure 1). 
Fig. 1 - Vue générale de la coupe stratigraphique et de la fouille à la fin de la mission (2014)

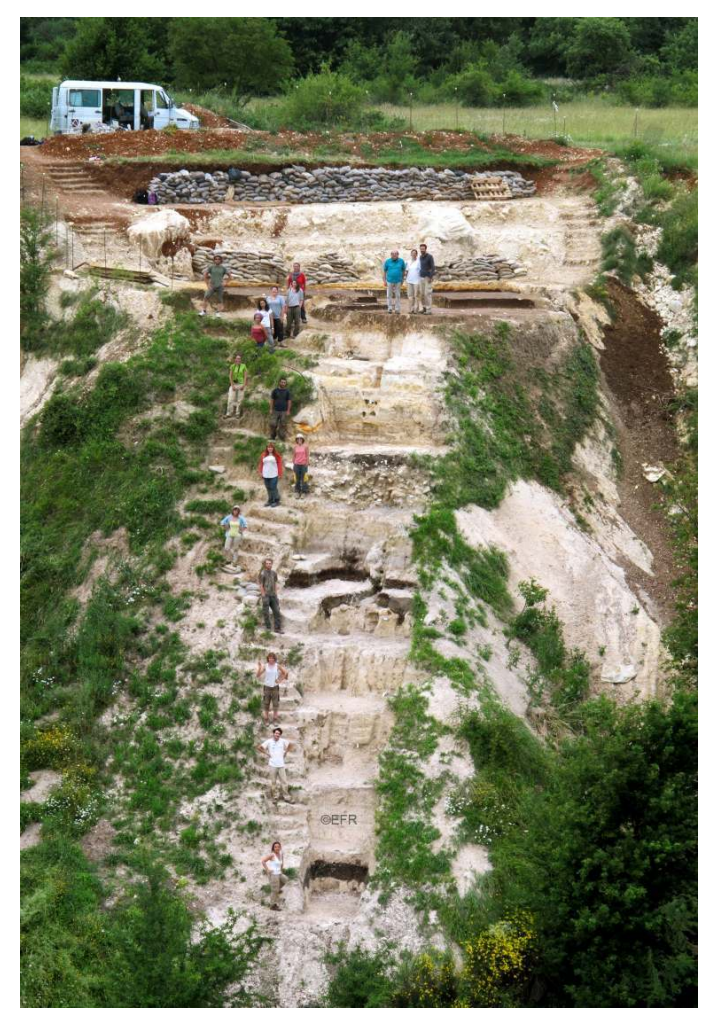

École française de Rome et E. Nicoud.

\section{Fouille archéologique}

\section{Déroulement}

5 L'objectif principal de la mission 2014 consistait à obtenir plus d'informations sur les niveaux d'occupation ALB et LDP, grâce à la fouille d'une aire suffisamment ample pour être significative (figure 2). En effet, durant la mission 2013, des zones de concentrations des vestiges lithiques et fauniques semblaient se distinguer, mais seule l'ouverture d'une surface plus grande pouvait permettre d'appréhender la répartition spatiale des vestiges. Souhaitant dans un futur proche, ouvrir une petite aire de fouille dans le niveau ABF situé à $5 \mathrm{~m}$ de profondeur, nous avons en priorité fouillé la zone proche de la ravine. L'aire de fouille du premier paléosol (niveaux ALB et LDP) situé entre 3,80 et 4,30 $\mathrm{m}$ de profondeur a été élargie, d'abord à l'aide de la pelle mécanique puis manuellement (figure 3). Le niveau ALB (argile limoneuse brune) est actuellement ouvert sur $48 \mathrm{~m}^{2}$. Après la mise au jour de la surface d'érosion irrégulière entre le niveau supérieur CGB et le toit de ALB, la fouille a débuté par passes de $5 \mathrm{~cm}$. Le niveau LDP (lit de pierres) sous-jacent, présente des caractéristiques physiques radicalement différentes de ALB ; il a été fouillé sur $22 \mathrm{~m}^{2}$ (figure 4). 171 artefacts en silex ont été découverts en ALB et 50 en LDP, dans un très bon état de conservation, 45 restes fauniques et 28 fragments de charbon en ALB (figure 5).

6 À la différence de l'an passé, les restes fauniques découverts cette année sont assez nombreux et parfois dans un bon état de conservation. L'une des découvertes les plus 
exceptionnelles est un fragment d'os portant de possibles traces de découpe, qui témoignent d'activités de boucherie.

Fig. 2 - Mise en sécurité des paliers en amont de la fouille et création de marches pour faciliter l'accès au site au début de la campagne.

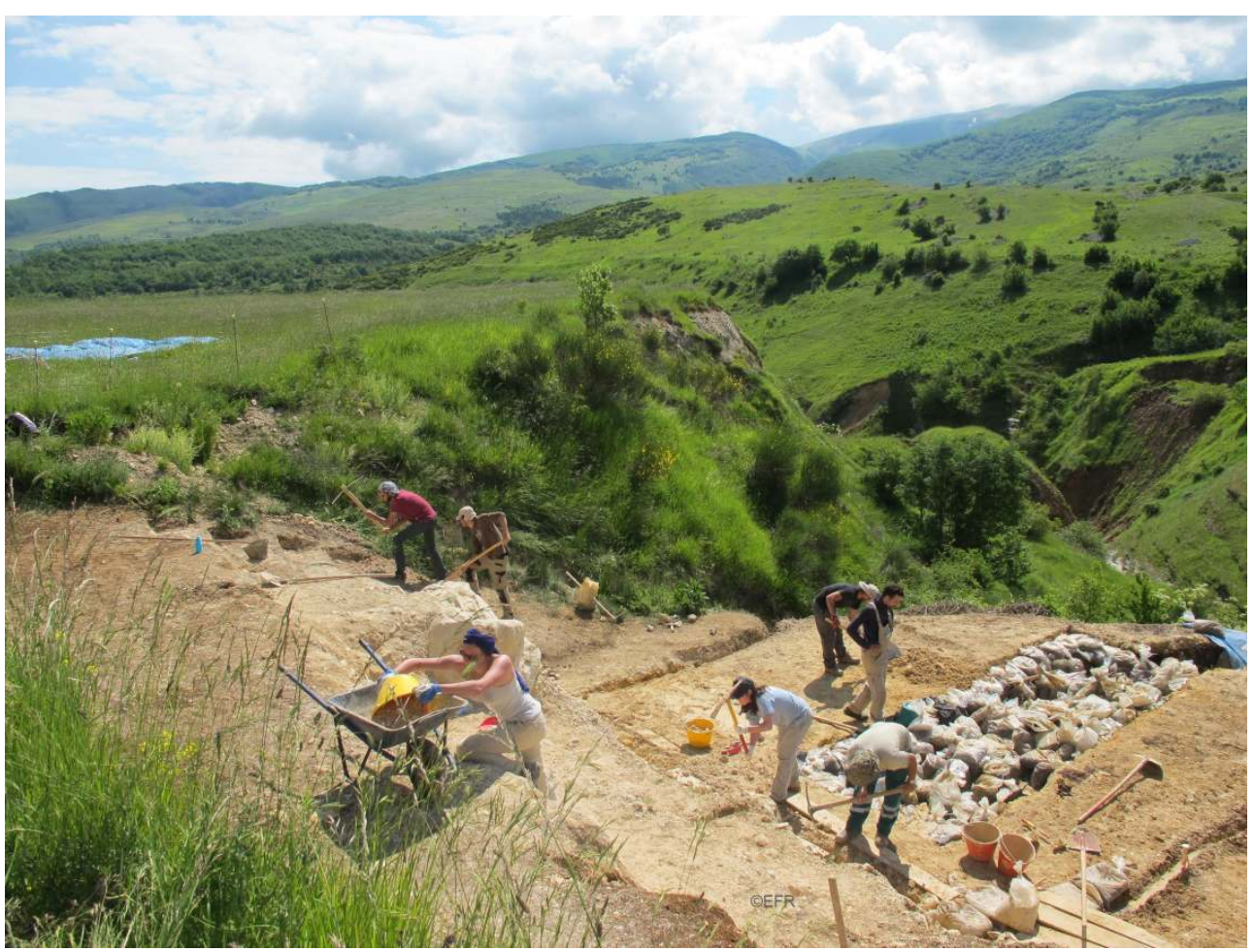

École française de Rome. 
Fig. 3 - Valle Giumentina 2014. Extension de l'aire de fouille en 2012, 2013 et 2014.

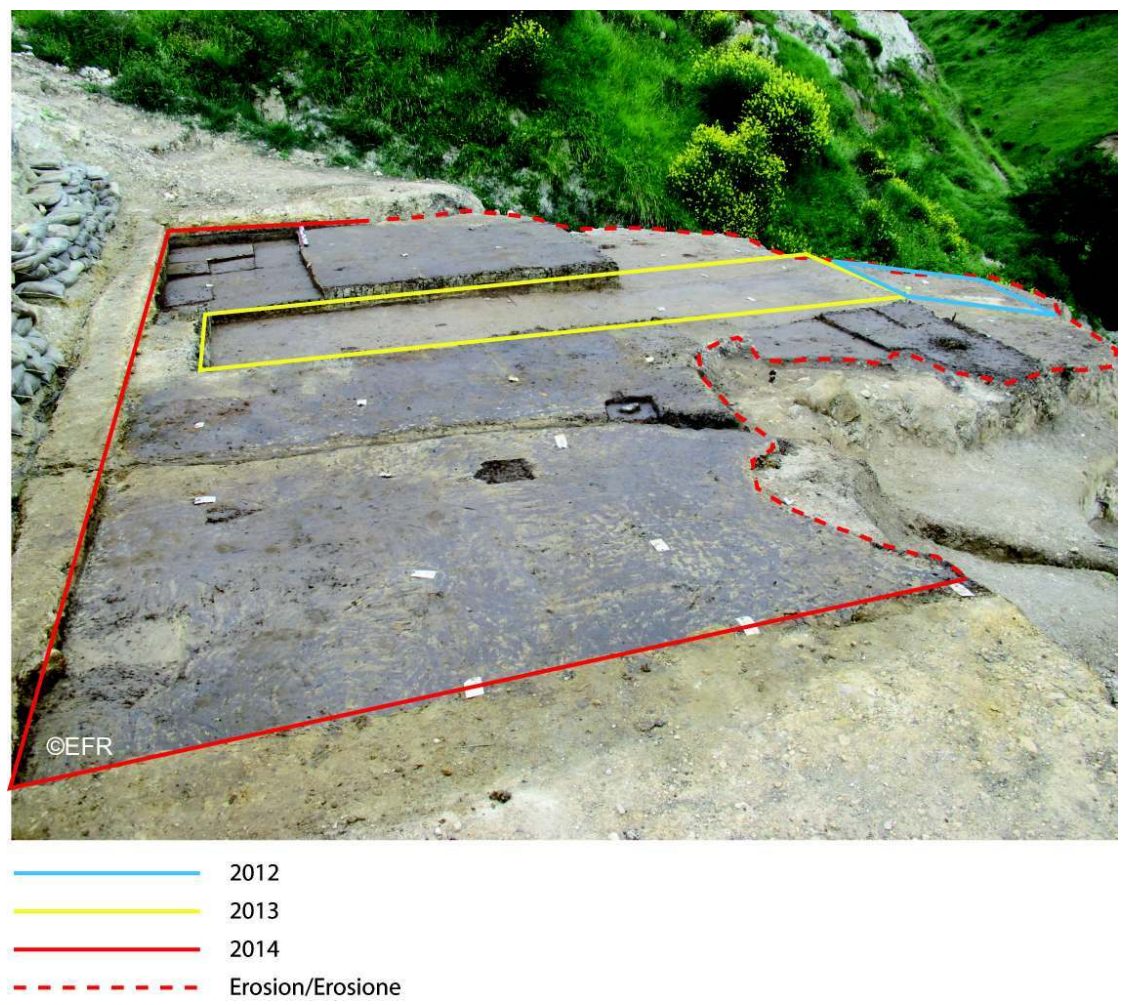

École française de Rome.

Fig. 4 - Valle Giumentina 2014. Surface du niveau LDP en cours de fouille.

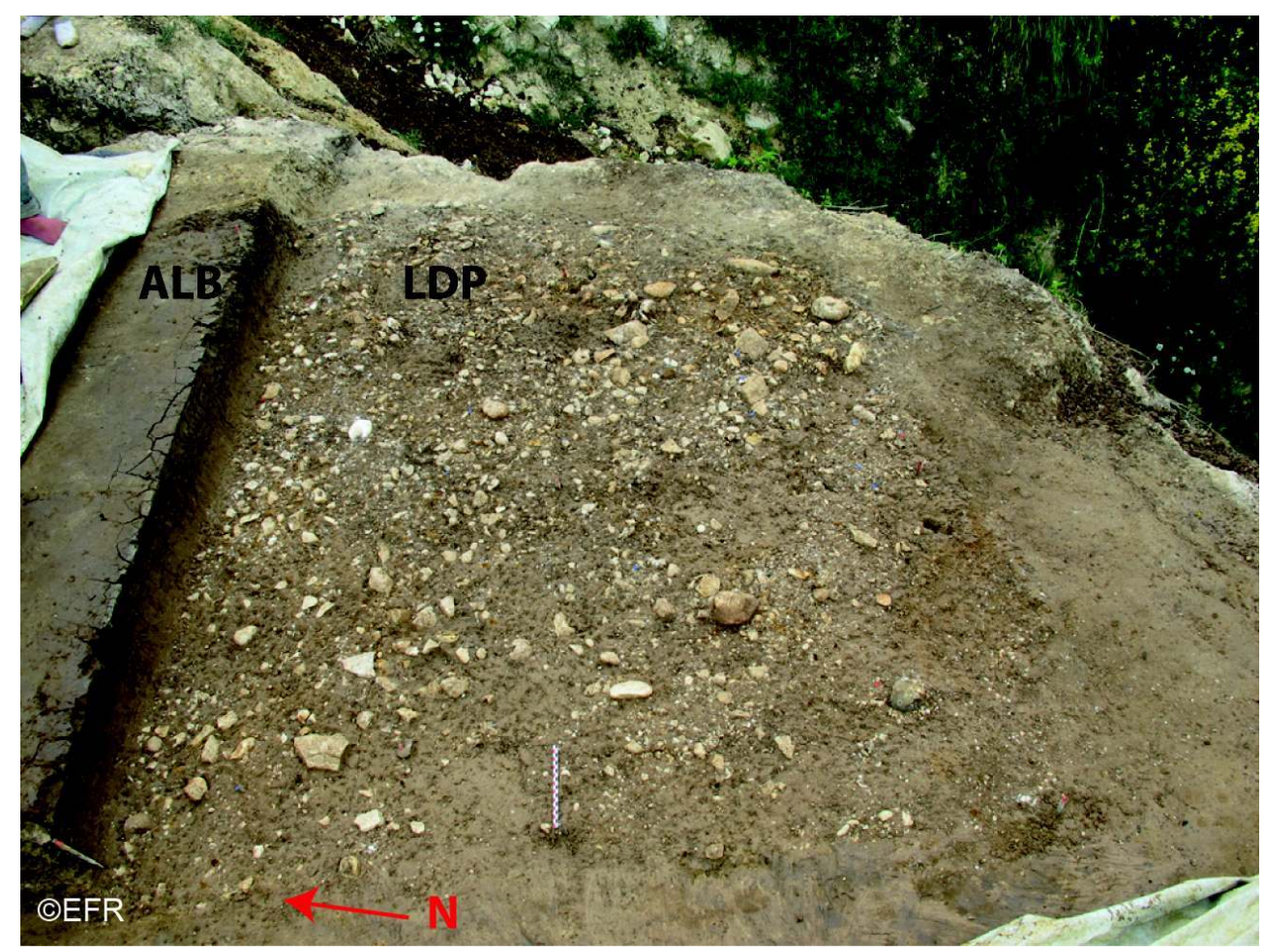

Le niveau ALB en cours de fouille, est visible à gauche de la photo. École française de Rome. 


\section{L'industrie lithique}

7171 artefacts en silex proviennent du niveau ALB et correspondent à diverses phases de la chaîne opératoire de production par débitage : il s'agit de 3 nucléus et pour le reste d'éclats et fragments d'éclats souvent partiellement corticaux, de dimensions variées, d'outils retouchés et de débris (tableau 1). Selon les premières observations, cette industrie à éclats est de conception abstraite ${ }^{2}$, à méthodes variées (SSDA ${ }^{3}$, tranches de saucissons, récurrent unipolaire). Les produits sont de volumes et dimensions variables. Des dos sont recherchés; ils peuvent être naturels, constitués d'une surface de débitage antérieure sécante, ou obtenus par fracture. Les supports sont parfois confectionnés par une retouche variée, scalariforme à marginale. Les objectifs fonctionnels sont pluriels et les tranchants multiples, depuis des bords rectilignes courts d'angle aigu ou des bords de $5 \mathrm{~cm}$ d'angle ouvert de près de $45^{\circ}$, des outils lourds avec des bords émoussés d'angle de $80^{\circ}$... Ces bords peuvent être par ailleurs denticulés et encochés. La distribution des vestiges n'est pas homogène et l'analyse préliminaire des plans montre que certaines zones sont riches d'artefacts lithiques et fauniques (figure 6). Toutes les observations effectuées sur le terrain seront complétées et approfondies durant des missions d'étude au cours de l'hiver 2014-2015 au niveau de l'étude tant technologique que tracéologique (A. Zupancich et C. Lemorini).

Tableau 1 - Décompte typo-technique de l'industrie lithique découverte à Valle Giumentina lors de la mission 2014.

\begin{tabular}{|c|c|c|}
\hline VG -INDUSTRIE LITHIQUE 2014 & ALB & LDP \\
\hline $\begin{array}{l}\text { Support non transformés } \\
\text { Nucléus } \\
\text { Éclats et fragments d'éclats }\end{array}$ & $\begin{array}{l}146 \\
2 \\
135\end{array}$ & $\begin{array}{l}37 \\
2 \\
30\end{array}$ \\
\hline Dont éclats & 35 & 5 \\
\hline Dont petits éclat $<20 \mathrm{~mm}$ & 20 & 3 \\
\hline Dont très petits éclats $<15 \mathrm{~mm}$ & 80 & 22 \\
\hline Cassons (fragments indêterminés) & 7 & 3 \\
\hline Bloc brut & 0 & 2 \\
\hline Galet calc aire & 1 & 0 \\
\hline Supports transformés & 25 & 13 \\
\hline Édats et fragments d'éclats & 24 & 12 \\
\hline Dont éclats & 20 & 9 \\
\hline Dont petits éc lat $<20 \mathrm{~mm}$ & 1 & 3 \\
\hline Dont très petits éclats $<15 \mathrm{~mm}$ & 3 & 0 \\
\hline Nucléus & 1 & 1 \\
\hline TOTAL & 171 & 50 \\
\hline
\end{tabular}

École française de Rome. 
Fig. 5 - Exemples d'industries lithiques et de restes fauniques découverts durant la fouille.
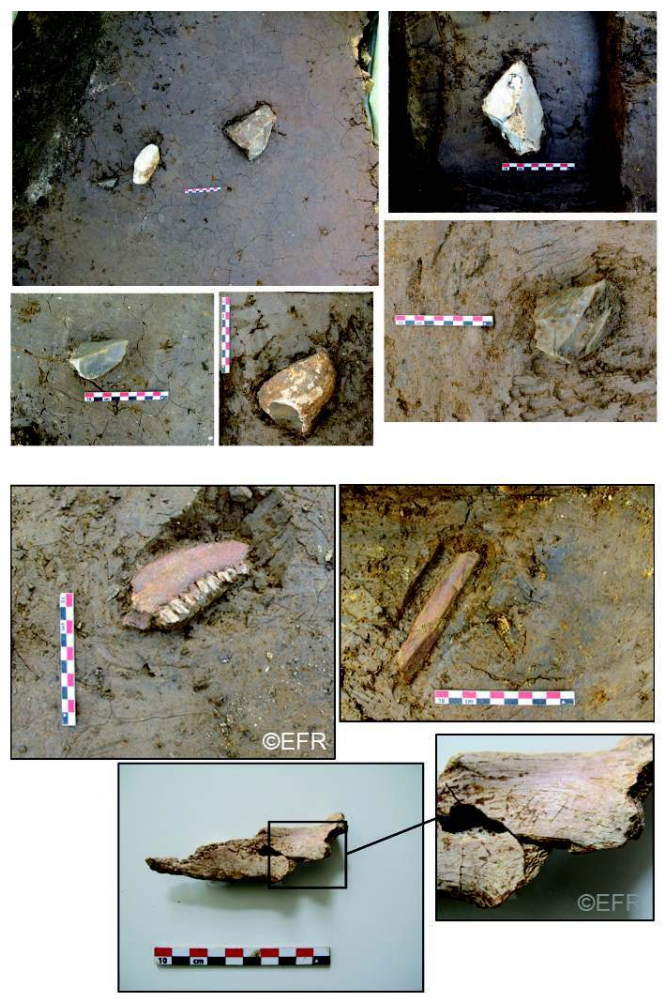

École française de Rome.

Fig. 6 - Plan de répartition des vestiges du niveau ALB.

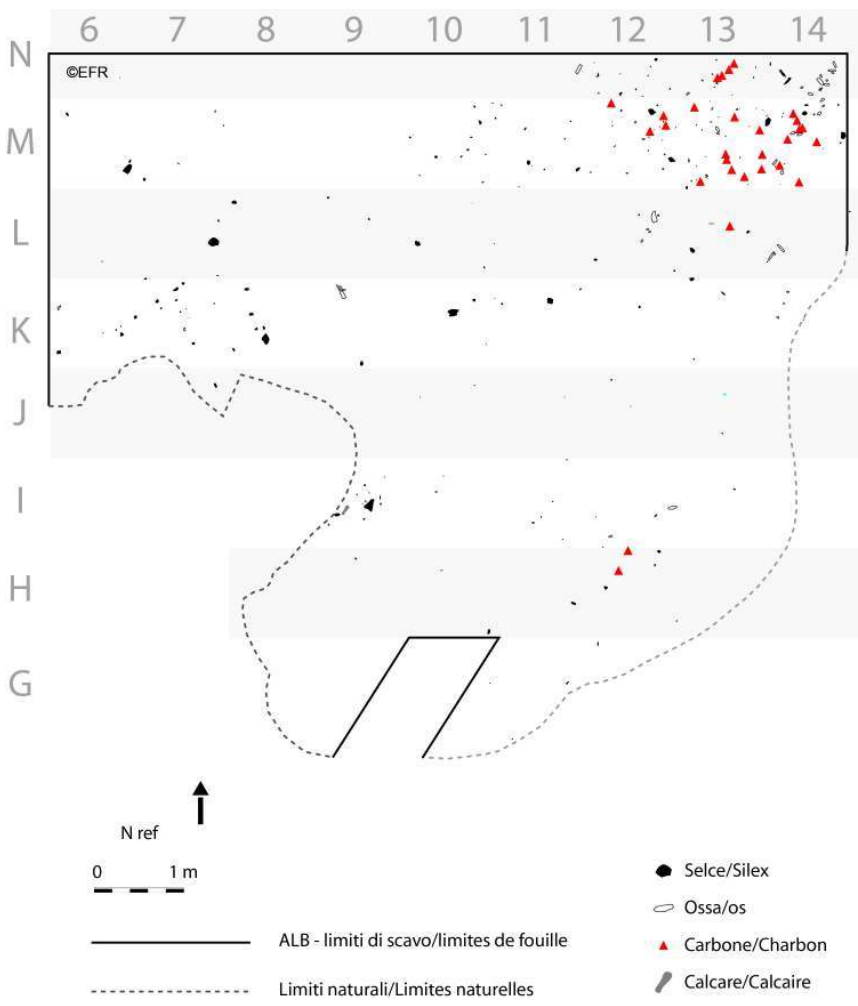

École française de Rome. 


\section{Les restes fauniques \\ (P. Mazza et M. A. Rossi)}

8 L'échantillon de restes faunique analysé par Paul Mazza et Maria Adelaide Rossi comprend seulement six restes déterminables aux niveaux anatomique et taxonomique ainsi qu'un proximal d'épiphyse d'un troisième métatarse droit. Ce sont des restes de Cervus elaphus exclusivement, provenant certainement du même individu. L'état d'usure de la molaire de la mandibule droite indique qu'il s'agit d'un adulte de plus de 3 ans. Quatre pièces provenant du même troisième métatarse gauche remontent. Des fragments d'un troisième métatarse droit sont présents. Quelques transformations anthropiques ont été observées. Il s'agit de traces de percussion et des stries et impacts visibles sur une vertèbre thoracique. Cette dernière pièce est particulièrement intéressante. Les surfaces articulaires caudales ont été percutées par un coup oblique à l'axe de l'épine dorsale. Sur le côté droit, des stries parallèles sont présentes, orientées dans l'axe du coup. L'épine dorsale est aussi érodée. L'apex est cassé et la fracture indique une fracturation de l'os sec. Le côté droit qui porte des stries est aussi érodé, faiblement, par les éléments météoritiques alors que les surfaces articulaires crâniales sont très érodées. En contraste avec le côté droit, le côté gauche ne semble pas érodé par les éléments météoritiques mais altéré par la corrosion du sol et les racines. Tout cela indique que cette pièce a été décharnée par l'Homme puis abandonnée, a reposé sur le substrat le côté droit exposé à l'air durant une longue période avant son enfouissement.

9 L'échantillon comprend aussi trois restes de micromammifères, dont deux incisives inférieures et un fragment de mandibule gauche. Ce dernier appartient à Microtus arvalis. Il contient une incisive avec $\mathrm{m} 1$ et $\mathrm{m} 2$. Le Campagnol commun habite les steppes et prairies sèches mais aussi les champs cultivés ouvert de type petits prés, champ de céréales et pâturages. Il peut vivre dans des conditions plus froides qu'aujourd'hui.

\section{Cadre chronostratigraphique et paléoenvironnemental}

\section{Données chronostratigraphiques}

Les dosimètres enfouis l'an passé dans le cadre des datations OSL ont été prélevés en début de mission (C. Lahaye). Les résultats devraient être disponibles au printemps 2015. La coupe stratigraphique VV1 a été prolongée de près d'un mètre (jusqu'à $17 \mathrm{~m}$ de profondeur), de façon à atteindre un niveau contenant des dépôts volcaniques, repéré lors de l'analyse de la carotte. Des prélèvements pour les datations Ar40/Ar39 ont été effectués en différents niveaux. Elles seront produites au laboratoire du CEA de Gif-surYvette (A. Peirera, S. Nomade). L'analyse isotopique des minéraux volcaniques se poursuit (B. Giaccio). Les résultats devraient être disponibles au printemps 2015. Une dent de cerf fait l'objet d'une datation ESR (J.-J. Bahain).

11 Dans le cadre de l'étude sédimentologique de la séquence sédimentaire de Valle Giumentina, une étude de l'anisotropie de susceptibilité magnétique (AMS) des sédiments a été initiée afin de préciser les paléodirections de transports particulaires existant au moment du dépôt (J.-Ph. Degeai). Cette technique, en mesurant la susceptibilité magnétique d'un échantillon dans différentes positions, permet de reconstituer l'ellipsoïde de susceptibilité magnétique du sédiment dont la direction d'élongation peut 
indiquer le sens du transport sédimentaire. Un total de 116 échantillons a été prélevé sur les coupes VV1 et VV2. Les échantillons, prélevés dans des pots cylindriques de D $25 \mathrm{~mm}$ x H 22 mm, ont été orientés. Une vingtaine d'échantillon a fait l'objet de mesures à l'aide d'un susceptibilimètre Bartington MS2, du capteur MS2B (mesure en HF 4,65 kHz), et du logiciel de traitement AMS-BAR v1.1. Pour chaque échantillon, la susceptibilité magnétique a été mesurée dans 18 directions en range 0.1, et trois réplicas ont été effectués. Des tests effectués sur des échantillons contigus issus d'une même couche mais prélevés selon des directions d'affleurements différentes montrent que les résultats obtenus sont trop sensibles à la direction de prélèvement. Ceci est vraisemblablement dû à la nature meuble et peu cohésive des argiles échantillonnées qui ont été déformées lors de l'introduction du pot de prélèvement dans le sédiment. La technique de prélèvement introduit donc un biais d'échantillonnage trop important pour que les résultats d'AMS puissent être interprétables. Compte tenu de ces résultats préliminaires peu concluants, il a été décidé de ne pas exploiter plus avant cette technique d'analyse.

\section{Études malacologiques}

\section{(N. Limondin-Lozouet)}

1256 prélèvements sédimentaires ont été réalisés pour l'étude malacologique. L'étude préliminaire de la malacofaune par Nicole Limondin-Lozouet donne des résultats intéressants.

Les mollusques terrestres et dulcicoles sont des organismes dont la distribution est dépendante des gradients de température et d'humidité de leurs habitats et sont inféodés à la végétation. Par ailleurs la plupart des espèces européennes sont de petite taille (inférieure à $1 \mathrm{~cm}$ ) et se déplacent sur de très courtes distances. Les mollusques sont par conséquent d'excellents indicateurs des biotopes locaux.

Les coquilles comportent divers caractères (forme, denticulations, ornementations, striation, etc.) qui permettent dans la plupart des cas une détermination au niveau spécifique y compris sur des spécimens fragmentés. Les malacofaunes continentales constituent donc un biomarqueur efficace pour la restitution des environnements passés et sont particulièrement sollicitées dans les environnements sédimentaires carbonatés où les coquilles se conservent très bien comme c'est le cas à Valle Giumentina. Outre la reconstitution des paléoenvironnements les mollusques peuvent aussi apporter des informations biostratigraphiques car beaucoup d'espèces ont eu une occurrence variable au cours du Quaternaire (changement d'aires de répartition lié aux cycles climatiques globaux) et certaines ont même disparues.

15 Sur le site de Valle Giumentina, l'opportunité d'étudier les malacofaunes est apparue particulièrement importante car aucun bioindicateur n'est préservé dans les dépôts de la coupe. L'étude malacologique est donc l'unique source possible pour apporter des éléments directs à la reconstitution des paléobiocénoses contemporaines de l'accumulation sédimentaire et en particulier des phases d'occupation humaine.

16 Le pas d'échantillonnage adopté est de 10 à $15 \mathrm{~cm}$ selon l'épaisseur des niveaux et le volume constant de 10 litres de sédiment par prélèvement. Dans un premier temps 64 échantillons ont été identifiés pour prélèvement depuis le niveau archéologique supérieur en cours de fouille jusqu'à la base accessible de la coupe, à $17 \mathrm{~m}$ (figure 7). Parallèlement à l'échantillonnage, les opérations de tamisage ont été mises en place afin 
de réduire le volume des échantillons et optimiser leur envoi au Laboratoire de Meudon pour analyse (figure 8). Cette double activité a permis de tester au fur et à mesure la teneur fossilifère des dépôts et d'identifier rapidement certaines passées stériles en coquilles, ainsi une douzaine d'échantillons correspondant à des dépôts sableux plus grossiers ont pu être éliminés d'emblée.

Au total l'échantillonnage de la coupe comporte 52 prélèvements, auxquels il faut ajouter un deuxième ensemble de 3 échantillons concernant le niveau archéologique supérieur, qui ont été réalisés postérieurement, sur une zone apparue plus riche en coquilles. Enfin un dernier prélèvement à été pris dans un niveau repère extrêmement fossilifère sur une coupe latérale à proximité du site, afin de vérifier sa concordance avec un paléosol identifié dans la partie supérieure de la séquence du site. Avec 56 échantillons c'est donc environ une demi-tonne de sédiment qui a été tamisée sur des de 500 microns afin de récolter la totalité des restes coquilliers et permettre une approche indispensable à la fiabilité des résultats.

$\mathrm{Au}$ cours du mois de juillet les échantillons ont été relavés au Laboratoire de Meudon et en partie triés sous loupe binoculaire pour récolter les coquilles.

Fig.7 - Échantillonnages et prélèvements malacologiques.

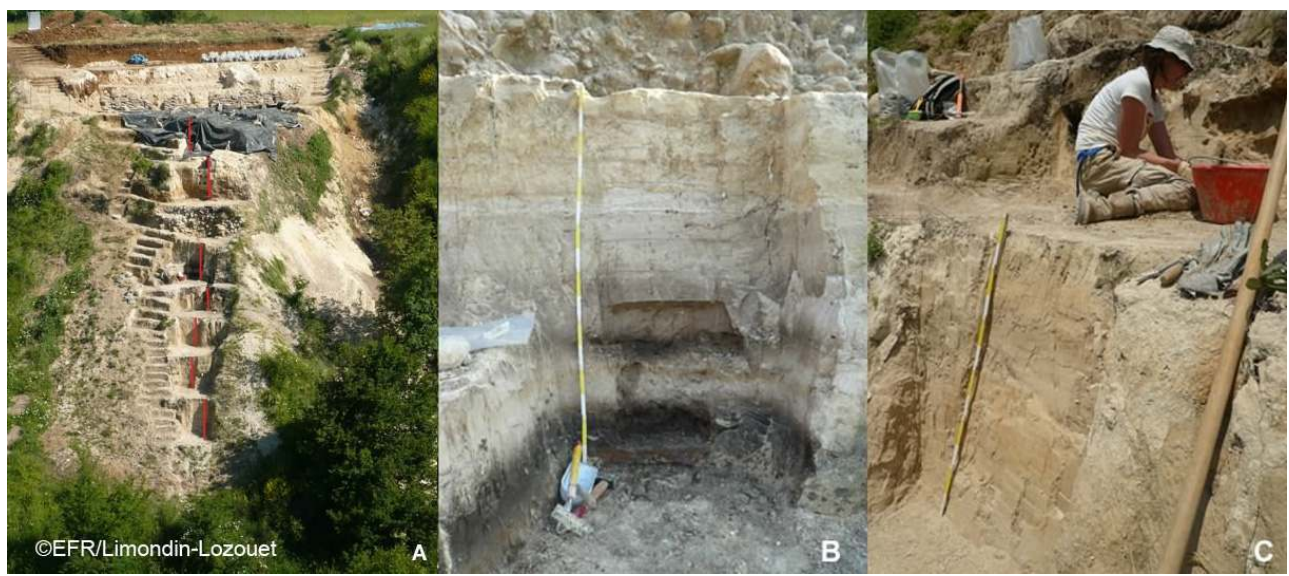

A. Échantillonnage malacologique de la coupe VV1 sur les différents paliers. B. Détail des prélèvements en colonne continue sur un pas de $10 \mathrm{~cm}$. C. Le volume constant est de 10 litres, il correspond à un seau par unité.

École française de Rome et N. Limondin-Lozouet. 

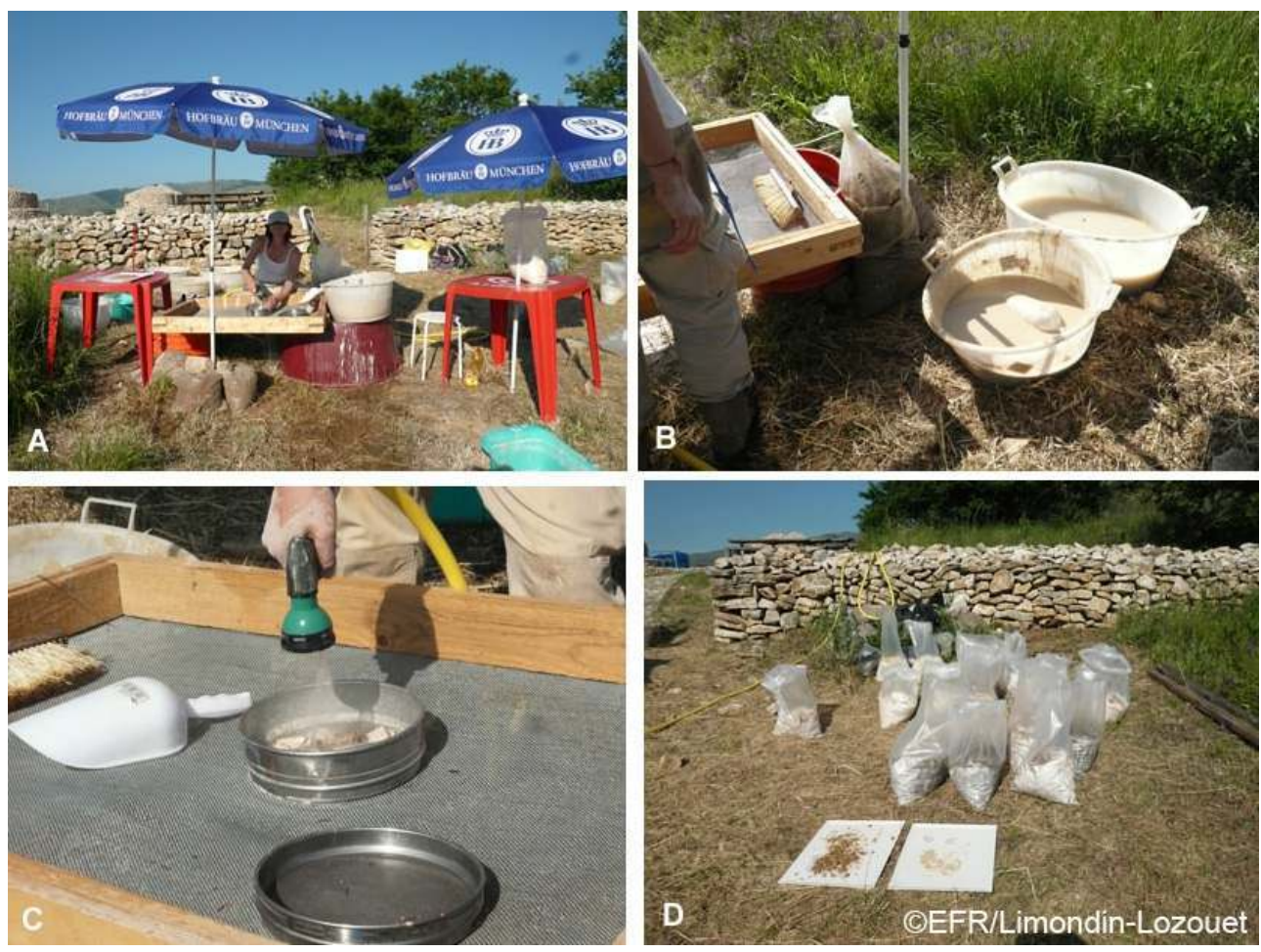

A. Poste de tamisage malacologique. B. Les sédiments prélevés sont mis à tremper. C. Le tamisage s'effectue sur une maille de 500 microns. D. Les résidus de tamis après séchage présentent un volume très réduits par rapport aux échantillons initiaux.

École française de Rome et N. Limondin-Lozouet.

19 À ce jour 32 des 56 échantillons ont été analysés. Les coquilles ont été identifiées puis comptées. Les échantillons traités ont été choisis de manière à couvrir l'ensemble de la succession stratigraphique pour obtenir rapidement une vision d'ensemble à affiner postérieurement. Certains problèmes de détermination sont encore à résoudre ou à préciser mais l'essentiel des espèces ont été reconnues et permettent de proposer une vue préliminaire sur les données environnementales enregistrées par les malacofaunes. Un premier constat porte sur la quasi absence des mollusques aquatiques dans les assemblages malacologiques. Localement l'environnement de la coupe de Valle Giumentina correspond à des biotopes terrestres, c'est-à-dire hors d'eau. Certaines passées sableuses sont cependant stériles en mollusques. Cette absence pourrait résulter soit de la destruction des coquilles soit de l'absence des faunes au moment du dépôt des sables. La première hypothèse est peu vraisemblable car les sables sont fins et ne peuvent être mis en cause comme agent mécanique de la destruction des coquilles. La seconde option, plus plausible, suggère que les niveaux sableux se sont accumulés rapidement sans laisser la possibilité aux faunes de mollusques de se développer. Ces épisodes sédimentaires pourraient alors correspondre à des phases d'inondation du site.

Les cortèges malacologiques terrestres font état de la succession de deux épisodes environnementaux bien contrastés qui renvoient à des ambiances climatiques nettement différentes. Dans la partie basale, entre $-11,5 \mathrm{~m}$ et $-7 \mathrm{~m}$ les mollusques indiquent la présence d'un milieu fermé de type forestier. De $-7 \mathrm{~m}$ au sommet du niveau archéologique supérieur la composition des assemblages change profondément, ils indiquent un paysage 
ouvert et sec. Parmi les espèces récoltées à Valle Giumentina il faut signaler l'occurrence de Jaminia malatest $a^{4}$ (figure 9). Cette espèce aujourd'hui éteinte, est endémique de l'Italie centrale et du sud, elle est caractéristique des dépôts du Pléistocène moyen et supérieur et constitue un indicateur de milieu froid et aride ${ }^{5}$.

21 Les résultats malacologiques sont encore à l'état préliminaire, il convient de terminer les analyses pour monter les diagrammes d'abondance et préciser d'éventuelles variations paléoenvironnementales plus détaillées. En l'état actuel des données, la malacofaune indique que les niveaux archéologiques se placent au cours d'une phase climatique froide durant laquelle le milieu apparaît ouvert, sec et hors d'eau.

Fig. 9 - Jaminia Malatestae (Esu 1988). Spécimen récolté à Valle Giumentina.

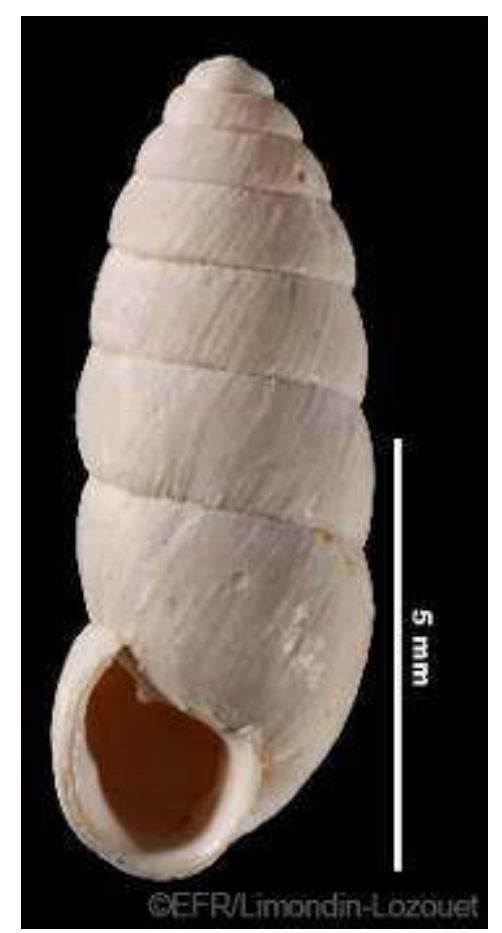

Espèce pléistocène éteinte, endémique de l'Italie centrale.

École française de Rome et N. Limondin-Lozouet.

\section{Études palynologiques \\ (F. Fusco)}

Parmi les analyses innovantes réalisées à Valle Giumentina, on compte aussi l'étude palynologique réalisée par Fabio Fusco. Ce type d'approche étudie les spores et les pollens, éventuellement conservés dans les dépôts sédimentaires. Ils permettent de retracer l'évolution de la végétation et par conséquence du climat, afin d'établir dans quel contexte environnemental les Hommes du Paléolithique ont peuplé le paléo-territoire de la Valle Giumentina.

23 Ainsi, le carottage mécanique effectué en 2012 et profond de $45 \mathrm{~m}$ a perforé l'entière séquence pléistocène, notamment sa partie lacustre. 300 prélèvements pour l'analyse pollinique ont été réalisés selon un pas de $10 \mathrm{~cm}$ dans les dépôts où la granulométrie était favorable (argiles et limons). 48 échantillons ont été analysés qui ont livré une flore bien 
caractéristique, indiquant un climat glaciaire, froid et particulièrement aride (figure 10). Aucune association typique de climat chaud ou tempéré n'est apparue. Il est ardu de savoir de quelle période glaciaire il s'agit. La difficulté résulte du fait que les périodes glaciaires ne se distinguent guère en matière de végétation, étant donné que sous ces conditions climatiques hostiles, seules peu de plantes peuvent résister (à condition qu'il n'y ait pas de couverture par la glace). Les interglaciaires quant à eux, sont bien caractérisés et caractérisables à partir de la végétation. Une autre difficulté augmente du fait de certains échantillons stériles ou avec peu de pollens, rompant ainsi la continuité de l'enregistrement. Toutefois, la découverte dans plusieurs cas de granules de Pterocarya, un type de noyer aujourd'hui disparu de la flore italienne et européenne, mais habituellement présente dans les bois collinaires jusqu'au Pléistocène supérieur, laisse penser que le « lac » de Valle Giumentina pourrait s'être formé avant le dernier glaciaire. De nouvelles analyses permettront d'éclairer l'âge de la formation lacustre.

Fig. 10 - Association pollinique de climat froid.
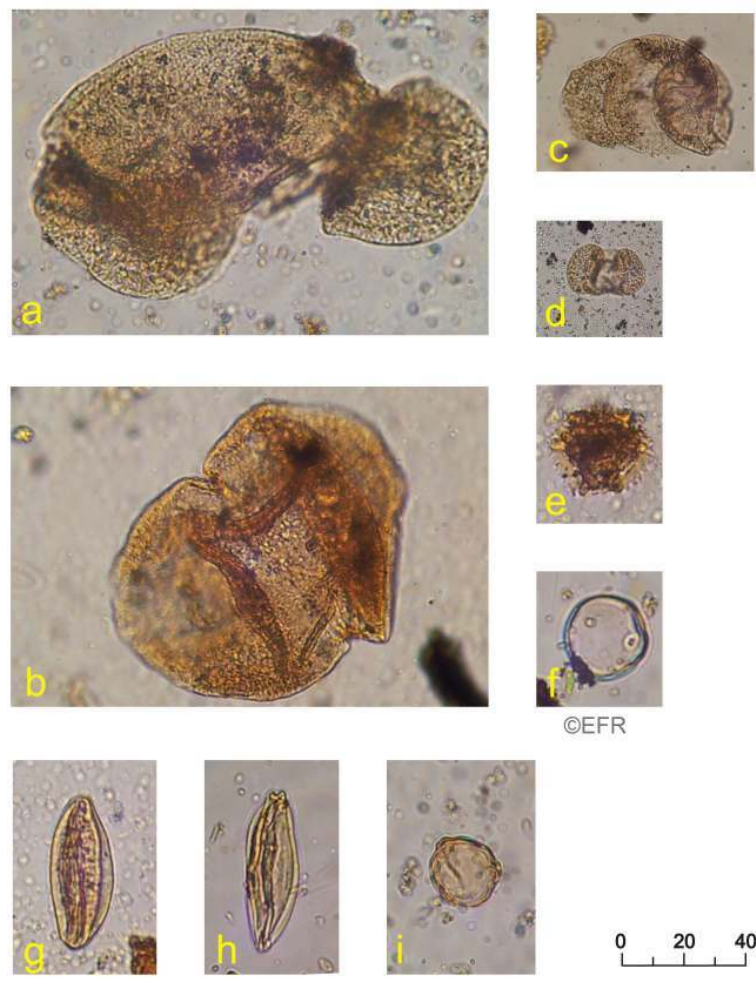

CEFR

La présence d'Ephedra, en deux formes indique des conditions arides. Cette association domine l'intervalle du sondage VG-1 analysé, suggérant une formation des dépôts lacustres en période glaciaire. a) Abies b) Picea ; c) Pinus ; d) Pinus tipo piccolo ; e) Asteraceae Cichorioideae ; f) Poaceae ( = Graminee p.p.) ; g) Ephedra t. distachya ; h) Ephedra t. fragilis, i) Betula.

Fabio Fusco.

\section{Reconnaissances géomorphologiques}

La mission de terrain reportée du 25 au 29 août et conduite par Valentina Villa et Christine Chaussé avait plusieurs objectifs. Tout d'abord, un relevé photographique complet de Valle Giumentina a été réalisé, couvrant le secteur qui entoure le site archéologique, jusqu'à la confluence avec le canyon de Santo Spirito/San Bartolomeo. 
Plus de mille photos ont été prises; grâce à la technique de photogrammétrie, elles permettront de construire un modèle numérique tridimensionnel détaillé de la vallée. En outre, la carte géomorphologique et géologique de la vallée a été réalisée; toutes les formations géologiques ont été cartographiées après avoir été reconnue en prospection pédestre. Enfin, en complément des prélèvements effectués sur la coupe et la carotte, des échantillons des formations repérées lors de ces prospections feront l'objet d'analyses sédimentologiques. Ces analyses supplémentaires permettront de compléter nos connaissances des modalités de mise en place des dépôts et de la chronologie de celle-ci à l'échelle de la Valle Giumentina.

\section{Perspectives}

Avant la prochaine mission, la majeure partie des données devrait être produites, tant en ce qui concerne le matériel archéologique issu des fouilles anciennes ou récentes, que des données géochronologiques et paléoenvironnementales (palynologie, malacologie, datations OSL, datations ${ }^{40} \mathrm{Ar} /{ }^{39} \mathrm{Ar}$, analyses isotopiques des téphras...). Les analyses sédimentologiques effectuées en laboratoire sont quant à elles presque terminées. Les corrélations stratigraphiques ont pu être effectuées entre les fouilles anciennes et actuelles, autorisant de replacer les vestiges dans le cadre chronologique et environnemental que nous nous efforçons de construire.

Aussi, la prochaine mission de terrain à Valle Giumentina sera essentiellement orientée vers la fouille des niveaux archéologiques (ALB, LDP, ABF). Nous nous laissons la possibilité d'ouvrir une autre aire de fouille en amont du ravin, où le niveau 37 des fouilles anciennes, c'est à dire le niveau à bifaces, serait facile d'accès.

\section{BIBLIOGRAPHIE}

Boëda 2013 = E. Boëda, Techno-logique \& Technologie : une paléo-histoire des objets lithiques tranchants, Prigonrieux [@rchéo-éditions], 2013.

D’amico - Esu 2011 = C. D'amico, D. Esu, Jaminia (Jaminia) malatestae Esu, 1988 (mollusca, gastropoda, enidae) from the Middle and Late Pleistocene of Central-Southern Italy. Palaeoecological implications, dans Il Quaternario, 24 (1), 2011, p. 67-74.

Demangeot - Radmilli $1966=$ J. Demangeot, A.M. Radmilli, Le gisement paléolithique de Valle Giumentina (Apennin central) et ses problèmes, dans Eiszeitalter und Gegenwart, 17, 1966, p. 159-299.

Esu 1988 = D. Esu, Jaminia (Jaminia) malatestae n. sp. from the Italian Pleistocene (Pulmonata : Enidae), dans Archiv für Molluskenkunde, 119 (4/6), 1988, p. 227-233.

Forestier $1993=\mathrm{H}$. Forestier, Le Clactonien : mise en application d'une nouvelle méthode de débitage s'inscrivant dans la variabilité des systèmes de production lithique au Paléolithique ancien, dans Paléo, 5, 1993, p. 53-82. 


\section{NOTES}

1. Demangeot - Radmilli 1966.

2. sensu Boëda 2013

3. sensu Forestier 1993

4. Esu 1988.

5. D'Amico - Esu 2011.

\section{INDEX}

institutions École française de Rome, Soprintendenza per i beni archeologici dell'Abruzzo, INRAP, ArScAn-AnTeT (UMR 7041), Università degli studi di Siena (preistoria e antropologia), di Pisa (biologia), di Firenze (scienze della Terra), di Roma La Sapienza, Université Paris 1 PanthéonSorbonne (ED de géographie de Paris), Université Bordeaux 3, CNR, CNRS (LGP UMR 8591, ASM UMR 5140, IRAMAT UMR 5060, LSCE UMR 8212 et HNHP UMR 7194), Max Planck Insitute for Evolutionary Anthropology, Muséum national d'Histoire naturelle, Commissariat à l'énergie atomique, Fondation Pescarabruzzo, Parco nazionale della Majella, Comune di Abbateggio, Museo delle Genti d'Abruzzo, Archeoclub di Pescara

Keywords : palaeolithic, Abruzzo, Middle Pleistocene, Valle Giumentina, lithic technology Mots-clés : paléolithique, Abruzzes, pléistocène moyen, Valle Giumentina, technologie lithique

\section{AUTEURS}

\section{ÉLISA NICOUD}

École française de Rome - elisa.nicoud[at]gmail.com

\section{DANIELE AURELI}

Università degli studi di Siena (U.R. Preistoria e Antropologia)

\section{MARINA PAGLI}

ArScAn-AnTET (UMR 7041) 